\title{
La teoría social urbana y el debate actual
}

\section{José Luis Lezama*}

El trabajo que aquí se presenta tiene por objetivo situar las aportaciones socioiógicas de la escuela ecologista clásica en el debate que actualmente tiene lugar, a propósito del objeto de estudio de la sociología urbana.

Esta escuela de pensamiento es rescatada como el primer gran intento por construir un objeto teórico para dar cuenta de los procesos sociales más significativos que tienen lugar en la moderna ciudad capitalista. Diversos autores, anteriores a los ecologistas, habían pensado la ciudad y su compleja problemática explicándola como una expresión (sociológica, económica, histórica, etc.) de las propias leyes de funcionamiento de la sociedad en la cual se insertaba. No obstante, los ecologistas trataron de demostrar, mediante una teoría específica de lo urbano, que la misma ciudad podría explicar todo un conjunto de conductas sociales que tenían lugar dentro de su dimensión ecológica.

En el momento actual, algunos de los paradigmas ecologistas siguen presentes, no obstante el significado sociológico que poseen no es el mismo. De esto dan cuenta autores que, como Lefebvre, Harvey, Massey y Giddens, han revalorado la dimensión espacial de los procesos sociales, volviéndola a colocar en el centro de la discusión sociológica.

\section{Antecedentes}

Entre 1915, fecha de la publicación del ensayo "La ciudad: propuestas para la investigación de la conducta humana en el medio urbano", de Robert Ezra Park, y 1938, año en que se publica el ensayo de Louis Wirth "El urbanismo como forma de vida", se asiste en la sociología estadunidense al proceso de construcción de un objeto teórico específico para una sociología específicamente urbana. Pero la ciudad y su compleja problemática social no sólo brindó el escenario para la elaboración de una sociología urbana, al mismo tiempo estimuló el surgimiento y la institucionalización de la más significativa tradición sociológica estadunidense.

La emergencia de problemas urbanos concretos debido al gran crecimiento de algunas ciudades estadunidenses de fines del siglo pasado y principios del presente, despertó un interés analítico especial por parte de estudiosos de diversas disciplinas de las cien-

* Profesor-investigador del Centro de Estudios Demográficos y de Desarrollo Urbano de El Colegio de México. 
cias sociales y no fue la llamada escuela ecologista clásica de Chicago $^{1}$ la única en tomar conciencia de esta problemática. En 1899, Adna Weber presentó una disertación en la Universidad de Columbia sobre el crecimiento de las ciudades en el siglo XIX. Durante los primeros años del presente siglo la antigua Chicago School of Civics and Philanthropy llevó a cabo una serie de estudios bajo la dirección de Edith Abbott y S. Breckenridge, en los cuales se analizaba a la población migrante (Burgess y Bogue, 1970:4) manejándose ya algunos de los conceptos que posteriormente se popularizarían en la literatura antropológica y sociológica dando lugar a la idea del llamado hombre migrante.

En 1915 la Sociedad Americana de Sociología llevó a cabo su primer congreso dedicado al campo de la sociología rural, y en 1925 se efectuó otro destinado exclusivamente a la sociología urbana con lo cual esta rama de la sociología adquiere, por decirlo así, su carta de ciudadanía en la comunidad científica estadunidense. En ese mismo año Burgess publica, bajo el título de The Urban Community, un libro que reúne los principales trabajos presentados en este congreso; allí se contiene desde algunos ensayos clásicos de Zorbaugh hasta los más novedosos de Wirth, pasando, por supuesto, por uno de los más sugerentes de Park; ${ }^{2}$ el artículo que cierra esta obra corresponde a uno de los fundadores de la sociología estadunidense, Sorokin. En ese año se publica también una de las obras más significativas y tal vez el primer gran clásico de los estudios urbanos en la sociología estadunidense: The City, de Robert E. Park, Ernest Burgess y Roderick McKenzie y en el cual se encontraban los diversos trabajos, publicados separadamente, de estos autores entre los años 1915 a 1925 (Hatt y Reiss,

\footnotetext{
${ }^{1}$ En los diversos trabajos que describen el surgimiento y consolidación de esta escuela, se coincide en incluir dentro de la escuela ecologista clásica de Chicago la obra de aquellos autores que publican sus trabajos en el periodo comprendido entre la primera y segunda guerra mundial, estableciendo como momento final de la etapa clásica de esta escuela de pensamiento el de la fecha de publicación del más sistemático de todos los trabajos comprendidos dentro de este enfoque, me refiero al libro de Amos Hawley; Human Ecology: A Theory of Community Structure de 1950. Otros autores señalan la fecha de los ensayos mencionados de Park (1915) y de Wirth (1938) para acotar el inicio y final del periodo de auge y consolidación de esta teoría. Todos coinciden en considerar como los clásicos de la Escuela Ecologista de Chicago a Robert Ezra Park (de hecho el fundador) Ernest Burgess y a Roderick McKenzie.

2 Me refiero a uno de los ensayos de este autor que sintetizan de manera clara y brillante las principales formulaciones del enfoque ecologista de lo urbano esto es: "The Urban Community as a Spatial Pattern and a Moral Order", que es incluido como introducción al libro The Urban Community.
} 
1957:3). En este periodo también surgen los trabajos sobre la comunidad de Lynds, los rigurosos estudios sobre sociología urbana y rural de Sorokin y Zimmerman y los clásicos manuales de sociología urbana de Bedford y Anderson (ibidem: 3).

\section{La comunidad y la sociedad como categorías analíticas}

En el esquema ecologista la ciudad y los procesos que en ella tienen lugar son explicados por dos tipos de legalidades teóricas. Por una parte, se tienen aquellos que emergen de las fuerzas competitivas, generando la estructura territorial, la disposición funcional del espacio urbano acotado bajo el concepto de ciudad. Por la otra, están los fenómenos que derivan de las características más particulares del hombre, de aquello que especifica al género humano distinguiéndolo del resto de los seres vivos, todo aquello que tiene que ver con la comunicación simbólica, el llamado libre albedrío y el conjunto de las instituciones que originan una tradición, una cultura.

Al primer orden de cosas corresponde el concepto de comunidad y al segundo el de sociedad. Es a esto a lo que se refiere Wirth cuando señala que la sociedad y los grupos sociales en general "contienen al menos analíticamente, dos órdenes: el que puede ser entendido como la comunidad y el que corresponde a la sociedad" (Wirth, 1964b: 168). Esto es así porque los hombres viven, por una parte, en un territorio físico y ecológico y, por otra, en un contexto determinado por relaciones psicológicas y sociales.

El ámbito de la comunidad, se refiere a aquel nivel existente en los sistemas sociales que reproduce el orden vigente del mundo natural. En este sentido las sociedades, al igual que los organismos vivos, organizan los actos de su vida colectiva por medio de un proceso de diferenciación que hace posible el desempeño de todas las funciones necesarias al sistema en su conjunto y el uso eficiente de sus recursos, en un mundo de relaciones y hechos que no sólo ocurren en el espacio, sino que también tienen una existencia temporal. A esta organización funcional instrumentada en el nivel biológico de la vida del hombre (dentro de un orden que reproduce el del mundo animal y vegetal), es a lo que los ecologistas llaman la división social del trabajo. En el sentido usado por los ecologistas este concepto no da cuenta del fenómeno social descrito por la sociología, sino de uno de carácter biológico que tiene más bien que ver con la organización funcional de una unidad ecológica vista desde la perspectiva de relaciones bióticas; por ello, Park y sus colegas hablan de una división del trabajo en 
el nivel analítico correspondiente a la comunidad; es decir, no de orden social sino natural.

En este nivel de la comunidad, los organismos llevan a cabo su vida en un mundo de mutuas relaciones y de funciones que se complementan estructurando, de esta manera, lo que se llama una verdadera cadena de la vida. Pero la vida social no siempre es vista como un conjunto de relaciones simbióticas y de buenos propósitos, como parecería denotarlo esta visión de seres que cooperan y se reproducen entre sí; es, al mismo tiempo, la expresión de una lucha intensa por la sobrevivencia en una sociedad y en un entorno natural connotado por la escasez de recursos y el afán incontenible de consumo que aparentemente caracteriza a los seres humanos. Bajo el condicionante de la escasez de recursos se desata una ardua batalla por la sobreviviencia en la que, de acuerdo con el esquema darwiniano, sobreviven los más aptos. La competencia, que permea todos los ámbitos de la vida social, se convierte en mecanismos de autorregulación mediante los cuales la comunidad, al tiempo que selecciona cuantitativa y cualitativamente a sus miembros, asegura su sobrevivencia y el acceso a estadios más avanzados de desarrollo.

Al margen de las muchas dimensiones de la existencia humana, los hombres, según la perspectiva ecologista, poseen también una dimensión animal y en ésta se pone de manifiesto lo que Wirth llama los efectos de su agregación física y de su hábitat (Wirth, 1964a: 178). Esto es lo que en la mayor parte de los trabajos de los ecologistas clásicos constituye el ámbito analítico del concepto de comunidad. Entendido como un "tipo ideal", el concepto de comunidad, enfatiza las relaciones simbióticas, la dimensión espacio-tiempo, la estructura física, la competencia y la división del trabajo de los miembros de un conglomerado humano (ibidem: 180).

Es éste el planteamiento analítico de Park, en el cual se expresan los fenómenos de la naturaleza, que le permiten descubrir rasgos invariantes en la estructura de la ciudad, como expresión de una naturaleza humana inmutable. Es esto, a su vez, lo que explica, según Park, los límites de la planeación urbana y de las acciones políticas que tienen como propósito corregir los problemas derivados de la aglomeración en las grandes ciudades. Estos límites en la regeneración de la estructura y del orden moral urbano deriva de las fuerzas inmutables que gobiernan todos los ámbitos de la vida, incluyendo la vida social. La ciudad que a Park interesa no es aquella que se manifiesta en una estructura social específica o bajo la forma de los fenómenos más típicamente sociales como son la estructura, la movilidad y el cambio social, entre otros, sino 
las expresiones territoriales de estos hechos. En la lucha que entablan los hombres por acomodarse a su medio ambiente se ven inmersos en un constante tránsito. Éste no sólo los reacomoda, en términos de su posición en la estructura social, sino también en la posición que ocupan en el territorio; es decir, en su localización (Park, 1968: 6). Pero esta localización, este lugar que ocupan en el medio ambiente que los contiene y este mismo medio ambiente en el que viven, como diría Hawley (op.cit.), en una asociación inevitable e indisoluble, provoca en los propios hombres la parte esencial de su conducta social y de su comportamiento colectivo.

Pero existe otro ámbito de la vida del hombre, uno que no está sometido al mundo de las pasiones y de los instintos. Éste es el de lo racional y del llamado libre albedrío. En este nivel de la realidad el hombre no sólo conforma un orden moral, que le da su especificidad humana, sino que este mismo orden moral termina por imponerse a los impulsos de su voluntảd animal, sometiéndolo al grupo social del cual emerge. El hombre deviene en ser social a fuerza de ir contra las expresiones de su libertad e interés individual, de aquello que los enfrenta en el plano de la competencia y de la lucha por la vida. En el plano de la sociedad los hombres se plantean la consecución de sus fines bajo la forma de la cooperación, por ello los otros no se presentan como enemigos sino como compañeros de equipo. Si en el ámbito de la comunidad la competencia era la fuerza que regía las relaciones entre los individuos y grupos sociales, en el de la sociedad la cooperación es la energía que impulsa la acción individual y colectiva. A diferencia de la comunidad, en donde la vida social se expresa a través de una estructura espacial, en la sociedad se hace por medio de un orden moral. La presencia de este orden moral dirige los actos individuales hacia la búsqueda del consenso y la concertación, pero no con el propósito de imponer el dominio de las voluntades individuales, sino para que éstas se organicen de acuerdo con la llamada conciencia colectiva, de acuerdo con el orden que emana del organismo social como un todo. El aspecto social, denotado bajo el concepto de sociedad, es entonces aquel que se manifiesta en la subordinación de los individuos a los fines sociales buscándose el principio de la estabilidad en contra de las fuerzas centrífugas que empujan hacia la desintegración.

Pero ¿qué es lo que convierte a una comunidad en sociedad?, ¿cuáles son los elementos que demarcan la frontera entre uno y otro territorio? $\mathrm{Al}$ respecto, Wirth señala que la diferencia entre el mundo de los hombres y el de los animales radica en la presencia, en el caso de los primeros, de la comunicación. En este orden de ideas los hombres realizan una vida comunitaria por las cosas 
que tienen en común, y éstas las adquieren por la vía de la comunicación. En el mundo de las plantas, señala este autor, basta con analizar la comunidad por medio de sus relaciones simbióticas y de la cooperación competitiva; en cambio, entre los hombres esto no es suficiente. Éstos deben ser analizados a través de "la participación de los individuos en empresas, esperanzas e ideales comunes y a través de los mecanismos de comunicación e interacción social que no radican en el organismo sino que, más bien, existen en el lenguaje, en los símbolos colectivos, en las leyes y costumbres; en pocas palabras, en la herencia social" (Wirth, 1964b: 168).

Pero el nivel de la sociedad, aun cuando sea el punto de partida y de llegada de los procesos de cambio en el interior de la comunidad, no se asocia necesariamente en la obra de los ecologistas clásicos con un estado de perfección en contraste con uno imperfecto que estuviera representado por la comunidad. En realidad, entre ambos niveles se produce una dinámica de complementariedad; de tal suerte que, aun en los momentos de más intensa competitividad y aun cuando las situaciones predominantes sean aquellas que derivan del despliegue de las fuerzas naturales más ciegas, esto no es sino la expresión por parte del sistema social, de sus mecanismos de autorregulación de tal manera que éste responda eficazmente a los cambios que tienen lugar en su interior y en su respectivo medio ambiente. Ésta es la forma en que la sociedad evoluciona hacia mayores grados de desarrollo accediendo a un nuevo y superior equilibrio social; es decir, a una nueva dimensión de aquello denotado bajo el concepto de sociedad.

La misma cooperación competitiva aparece precisamente cuando los competidores toman conciencia de los peligros que representa para la comunidad la confrontación extrema de sus miembros, por ello se plantean la necesidad de limitar y reglamentar sus conductas (Anderson, 1965). Visto de esta manera la cooperación no es sino un momento de la competencia, aquel en el cual el sistema social reflexiona, por decirlo así, sobre sí mismo, capitalizando los logros del proceso de selección natural, y estableciendo las nuevas reglas de la convivencia y del consenso social.

Esta funcionalidad del cambio que tiene lugar en el nivel de la comunidad y que conduce el rumbo de la vida social hacia el nivel de la sociedad, lo expresa Park a través de las llamadas crisis ambientales.

Para la escuela ecologista existe en las comunidades una tendencia natural que las mueve hacia la búsqueda del equilibrio. Pero esto no impide que recurrentemente se encuentren en un estado de inestabilidad y de cambio. Las comunidades, además de su ubicación en el espacio, poseen una ubicación temporal. Ésta 
las hace susceptibles a las transformaciones que tienen lugar en las condiciones ambientales que las rodean y que constituy en su hábitat. Los cambios no sólo ocurren en el clima y el suministro de alimentos, sino también en las mismas posiciones que ocupan los distintos miembros de la comunidad. Al modificarse estas circunstancias, las comunidades enfrentan situaciones de desbalance en sus estructuras que las enfrentan a las mencionadas crisis ambientales (Bardo y Hartman, 1982).

La comunidad se encuentra ante una situación de crisis cuando se rompe el equilibrio existente entre recursos naturales y población. Bajo esta circunstancia los hombres (de la misma manera que los animales y las plantas), se hacen más competitivos, de tal suerte que la comunidad pueda acceder a una forma más evolucionada de la división del trabajo, esto es, una diferenciación más amplia en donde el surgimiento de nuevas funciones responde a la mayor complejidad de la vida comunitaria y, sobre todo, donde la propia comunidad se prepare para esa nueva etapa de su desarrollo que ha originado, precisamente como el resultado del despliegue de todas las potencialidades individuales puestas de manifiesto en el proceso de selección operado vía la competencia. La comunidad, al final de este periodo de cambios, entra de nueva cuenta en una etapa en la cual la competencia (símbolo del dominio de las fuerzas naturales), es sustituida por la cooperación (en la cual se impone el reino de la racionalidad, de la vida social).

En el esquema ecológico de Park la competencia y la cooperación constituyen dos fuerzas opuestas que guían la acción humana. En los momentos de mayor estabilidad, cuando las comunidades han recuperado su equilibrio, luego de las fuertes sacudidas a que las someten las crisis ambientales y cuando la cooperación ha ocupado el lugar de la competencia bajo sus formas más álgidas, o cuando las fuerzas bióticas disminuyen y los hombres viven su vida social bajo la forma de una competencia atenuada, así como de un reforzamiento de los fines colectivos, es cuando las comunidades humanas alcanzan el carácter de sociedad, y esto significa que han llegado a un nuevo equilibrio, el cual es susceptible de ser roto por las crisis ambientales subsecuentes (Park, 1988).

En este mismo esquema, según los ecologistas, las sociedades instrumentan sus propios mecanismos de perpetuación, de tal manera que puedan resistir las embestidas cíclicas de las crisis ambientales y de las fuerzas bióticas que anidan en la comunidad y que, finalmente, socavan el orden vigente y dan lugar a los cambios sociales. Estos mecanismos que empujan hacia el reforzamiento del orden social, atenuando en la medida de lo posible la competencia, constituyen la esencia y la razón de ser de este nivel 
de análisis comprendido en el concepto de sociedad. Éste es el dominio de las instituciones, de las costumbres, de las tradiciones; es decir, de ese producto que constituye el mayor nivel de especificidad de la esencia humana, la cultura (Bettin, 1982).

Pero el orden de fenómenos acotados en el nivel de la sociedad no constituye, al menos en los ecologistas clásicos, el ámbito de análisis de la ecología humana, sino más bien aquel comprendido en el de la comunidad.

Si en el modelo ecologista aparecen las fuerzas naturales que rigen en el ámbito de la comunidad como las que en verdad determinan la estructuración del orden urbano, es porque las leyes de la competencia que dominan en ese nivel de la realidad influyen también, de alguna manera, en los procesos que se presentan en el ámbito de la sociedad. Esto puede verse claramente cuando se analizan fenómenos como la cooperación (que corresponde al ámbito de la sociedad) los cuales, según el modelo ecologista, no tiene un fin por sí mismo, o uno que estrictamente conduzca hacia la búsqueda del consenso. En realidad, la cooperación también es vista como una de las estrategias que utilizan los miembros de la comunidad a fin de optimizar sus esfuerzos en la lucha de todos contra todos para proveerse de los ya de por sí escasos recursos urbanos. En el nivel de la sociedad, lo que en verdad tiene lugar es una sublimación de las formas de la competencia más animal, pero ésta no deja de existir y rige de todas maneras con todo su peso el orden de la vida social. Es esta circunstancia lo que explica que para algunos autores, la cooperación y los sistemas de alianzas que se establecen en el nivel de la sociedad sean, una forma encubierta de la competencia: "la gente hace alianzas para competir mejor, por tanto es el conflicto y no la cooperación lo que los ecologistas ven como la base de la organización humana" (Bardo y Hartman, op.cit.).

El orden social urbano que interesa a los ecologistas es aquel que deriva de la intensa competencia que se lleva a cabo entre los distintos actores que dan cuerpo a la sociedad urbana. La ciudad emerge de la concurrencia de los hombres en el espacio urbano y de su lucha por buscar acomodo en una sociedad que basa la fuerza de sus estructuras en la selección de sus miembros más aptos.

Por último, debe destacarse que estas distinciones analíticas entre la comunidad y la sociedad están construidas bajo la perspectiva de "tipos ideales", mediante los cuales se preténde sintetizar los aspectos esenciales de la vida social. Esto significa que estamos en realidad ante la construcción de un objeto teórico con el cual se pretende dar cuenta de fenómenos que en la realidad no guardan este mismo estatuto. En los hechos los fenómenos socia- 
les entre ellos los que ocurren en el medio urbano, no se presentan escindidos de manera tajante. Por lo tanto, el mundo de las relaciones simbióticas, de la estructura física, la competencia y la división del trabajo (propios de la comunidad) no se presenta necesariamente separada del de la comunicación, las normas, el consenso, los valores, el control social consciente y la acción colectiva (Wirth, 1964a) (que corresponden al nivel de la sociedad). La comunidad, como lo señala Wirth, también es sociedad y todas las sociedades tienen un orden de cosas que corresponden a la comunidad.

\section{La ciudad en el esquema ecologista}

\section{Estructuración física y social del espacio urbano}

El marco analítico utilizado por la escuela ecologista para dar cuenta de las fuerzas que operan en la conformación del orden urbano puede ser resumido en el planteamiento de McKenzie (op. cit.: 63-64), según el cual, el objeto de estudio de la ecología humana lo constituyen las relaciones espaciales y temporales de los seres humanos afectados por las fuerzas selectivas, distributivas y acomodativas en el medio ambiente. En este contexto uno de los conceptos que explica la conformación del espacio y de los procesos urbanos es el de la diferenciación, con el que la biología alude a la manera como los organismos vivos "se ven obligados" a adecuarse $^{3}$ a los cambios que tienen lugar en su medio ambiente. El incremento demográfico aparece dentro de la perspectiva ecologista como uno de los elementos que posibilita la diferenciación y su consecuencia más evidente es el cambio social. Esto es visto así porque la presencia de nuevos miembros en la comunidad rompe el equilibrio en el sistema social, en la medida que altera la relación de correspondencia entre población y recursos. En este contexto la pérdida del equilibrio es concebida no sólo como una cuestión normal, sino incluso necesaria, porque mediante ella la lucha competitiva adquiere mayor intensidad. Ésta desencadena el mecanismo selectivo que permite al organismo social escoger a sus mejores hombres y, de esta manera, fortalecer las estruc-

\footnotetext{
${ }^{3}$ La vida, señala Hawley, es una síntesis del organismo y del medio ambiente. El medio ambiente es el ámbito en el cual existen los organismos y constituye la materia prima de la vida. (Hawley, 1950: 12).
} 
turas sociales llegando, de nueva cuenta, a un nuevo y superior estado de equilibrio. En este contexto la diferenciación social es, por una parte, el proceso mediante el cual emerge el orden social y su expresión territorial y, por otra, es el mecanismo que desencadena el cambio implicando, además de la adaptación de los hombres a las modificaciones de sus ambientes, la superación del organismo social.

La ciudad vista a través de este esquema de estructuración y desestructuración, sustentado en la diferenciación funcional de la vida y las estructuras urbanas, es presentada desde distintos ángulos por los ecologistas clásicos. Burgess, por ejemplo, presupone un proceso de conformación del espacio urbano con base en este marco de diferenciación funcional, utilizando los conceptos de expansión, sucesión y concentración. En este sentido la diferenciación, mediante la cual emerge el orden urbano, se concretiza en su conocido esquema de los círculos concéntricos. Éste es manejado como un tipo ideal al cual no corresponde, por tanto, un referente empírico concreto, expresándose, en los casos estudiados por los ecologistas, bajo la forma de rasgos y tendencias generales que se aproximan en mayor o menor medida al modelo de referencia.

En un primer momento (círculo central del esquema) la ciudad, como respuesta al crecimiento físico y poblacional, desborda la primera zona de asentamiento en cuyo interior está contenido el distrito comercial central. Como resultado de esto, ocurre un primer momento de la diferenciación que da lugar a la llamada zona de transición (zona II) en la cual se asienta la industria ligera y negocios pequeños. La conformación de esta zona empuja a sus antiguos moradores, los obreros, hacia una tercera zona en la cual se instalan, huyendo de las áreas deterioradas y con el propósito de ubicarse cerca de sus fuentes de trabajo. Posteriormente se forma una zona IV que se caracteriza por viviendas independientes y departamentos para las clases acomodadas. Por último, el proceso de expansión y diferenciación da lugar a los llamados suburbios (zona V) o ciudades satélites.

La ciudad emerge de este proceso de expansión y diferenciación. Todo crecimiento en las dimensiones urbanas originales es correspondido por el surgimiento de un área nueva y funcional. La ciudad parece adecuarse de manera natural a su mayor tamaño y complejidad, no sólo creando áreas sucesivas de asentamiento, sino también generando mecanismos de adaptación social, creando individuos e instituciones que efectúen las cada vez más complejas formas que asume la división social del trabajo.

Pero la expansión no se reduce necesariamente a los procesos 
de extensión y sucesión, ella genera al mismo tiempo otro de concentración y descentralización, además de provocar transformaciones en la organización social. La concentración-descentralización se presenta porque, por una parte, en el centro de toda ciudad se concentran las actividades económicas, políticas y sociales que rigen la vida urbana y la vida social en general y porque, además, la descentralización se traduce en la formación de subcentros comerciales, los cuales tienen como característica su dependencia con respecto al gran centro comercial y financiero de la gran ciudad. A esto es a lo que Burgess llama la formación de un sistema de descentralización-centralizado (Burgess, op. cit.: 53-54).

Dos aspectos deben ser resaltados en este contexto, a propósito de la estructura física y social del espacio urbano. En primer lugar que esta concentración-descentralización expresa una necesidad funcional de las fuerzas económicas y políticas que actúan en el ámbito territorial de lo urbano, en el sentido que requieren un esquema de reproducción social basado en la centralidad (Gottdiener, op. cit.). En segundo lugar destaca, en este mismo esquema, la forma en que se utiliza el concepto ecológico de dominancia para explicar el fenómeno de la centralidad. En la ecología animal y vegetal, este concepto denota la presencia de especies dominantes dentro de un hábitat determinado. En la ciudad, las actividades económicas dominantes, están representadas por la industria y el comercio, las cuales, en el plano de la lucha competitiva, tratan de ubicarse dentro de las zonas estratégicas del centro. El principio de dominación surge, tanto por la importancia que poseen estas actividades económicas, como por el establecimiento de un patrón de precios del suelo que deriva de la presión ejercida en la búsqueda de espacios disponibles en las zonas que garantizan mayores ventajas económicas, resultando de esto una revaloración del precio del suelo en el centro de la ciudad. A partir de los precios del suelo del área central, se establece un gradiente que explica los de cada una de las áreas sucesivas que conforman el espacio urbano, surgiendo así un patrón de usos del suelo para distintos grupos funcionales. De esta manera la ciudad y su patrón ecológico surgen de la lucha de las instituciones comerciales e industriales por localizarse estratégicamente en el entorno urbano (Saunders, op. cit.).

El proceso de diferenciación aquí descrito se traduce también en uno de segregación mediante el cual los individuos y los grupos se distribuyen y se integran socialmente, con el propósito de emprender los distintos aspectos de la vida urbana a través de roles específicos en los cuales se materializan las normas y valores sociales. La diferenciación y segregación a través de los cuales la 
sociedad urbana responde a las necesidades de su propio crecimiento, origina, por una parte, la división social del trabajo (en el sentido ecológico), con su subsecuente estructura espacial y, por otra, la división en grupos sociales con su resultante estructura social.

Es en este sentido que el proceso de diferenciación expresado en el desarrollo de la comunidad en general y de la ciudad en particular, implica uno de selección y segregación física y social que genera, en consecuencia, las áreas y los grupos naturales.

Cada una de las zonas que emergen de la expansión urbana se va caracterizando por un tipo particular de grupo social; desde los más pobres (migrantes de reciente arribo) que ocupan el centro y la llamada zona de deterioro, hasta los sectores más opulentos que ocupan las zonas residenciales del exterior. Cada una de estas zonas se caracterizan también por conductas sociales específicas que van desde las patológicas (crimen, vicio, desintegración familiar) de los tugurios, hasta las más sofisticadas de las zonas ricas que simbolizan el éxito y el prestigio social. ${ }^{4}$ Por lo tanto, la ciudad no está únicamente constituida por un espacio físico, es además un entorno humanizado por la cultura de sus habitantes, naciendo así cada una de las zonas, de los barrios que la integran. De este modo - señala Park-, lo que en un principio es únicamente una expresión geográfica, se convierte en un barrio; es decir, en una localidad con sentimientos, tradiciones e historia propia (Park, 1968).

En su explicación del orden urbano, McKenzie también recurrió al esquema ecológico del mundo animal y vegetal. Para ello se valió de los conceptos de invasión, competencia, sucesión y acomodación, los cuales le permitieron exponer la forma en que los diversos grupos étnicos, así como las funciones económicas, se acomodaban en el territorio urbano. De esta manera, las áreas funcionales y culturales de que se compone la ciudad, son el resultado de un continuo proceso de invasión y acomodación (Gottdiener, op. cit.).

Todo aumento en la dimensión cuantitativa de la comunidad desemboca en un aumento cualitativo y ambos fenómenos desencadenan un proceso de diferenciación y segregación. En este sen-

${ }^{4}$ Gottdiener señala que el planteamiento de Burgess sobre la ordenación territorial a partir del esquema de los círculos concéntricos, deriva también en la propuesta de un "gradiente de patología social" que se hace patente en una ordenación por zonas de fenómenos como el crimen, la delincuencia, la enfermedad, etc. Éstos parecerían tener una mayor presencia en los asentamientos pobres del centro y una menor en los barrios exclusivos de las afueras (op. cit., 54-56). 
tido, el mismo proceso explicado por Burgess con su teoría de los círculos concéntricos, es visto por McKenzie como una constante expansión que provoca que, al sobrevenir el incremento demográfico, las casas-habitación, las escuelas y la iglesia, por poner algunos ejemplos, se esparcen en dirección contraria al centro, en tanto que éste es ocupado por las actividades comerciales y financieras en general. En la medida que continúa aumentando la población, la diferenciación también aumenta, apareciendo nuevos servicios y generándose una lucha competitiva por el espacio que se convierte en un elemento estratégico para la localización de las actividades comerciales en el centro y de los asentamientos residenciales en las afueras. La ciudad, por ejemplo, crecerá en una secuencia "sucesional" en el sentido de que el surgimiento de determinadas empresas y servicios está en función del cumplimiento de determinadas etapas (sucesivas) de desarrollo.

De la misma manera que en las formaciones vegetales, el llamado proceso sucesional resulta de un proceso de invasión secuencial. De manera similar también estas invasiones originan, en el caso de la comunidad humana, las formaciones, las segregaciones y las asociaciones.

Por medio del fenómeno de la invasión la ciudad no sólo estructura o reestructura su territorio sino tambien lo hace con las funciones urbanas que le dan vida a los procesos sociales que allí tienen lugar. McKenzie distingue dos tipos de invasiones: a) aquellas que resultan de cambios en el uso del suelo, y b) las que sólo producen cambios en el tipo de ocupante. En el primer caso se comprenden los cambios de un uso general a otro, como es el caso de una zona de uso residencial que cambia a uno comercial o industrial. En el segundo caso se comprenden todos los cambios de género en un área de uso particular, como son los económicos y raciales en los vecindarios.residenciales o en el tipo de empresas de servicios en una sección comercial.

Ahora bien, una invasión se produce con mayor o menor éxito, o con mayor o menor facilidad, en función del tipo de invasión y del grado de solidaridad de los miembros de la comunidad. En este sentido McKenzie señala que es de esperarse que el invasor indeseable tenderá a ubicarse en el centro industrial, ${ }^{5}$ en relación con las zonas residenciales, porque en el primero no encontrará

\footnotetext{
5 Las zonas de deterioro aledañas al centro son de fácil penetración porque allí se localizan los migrantes recién llegados entre los cuales no existen lazos profundos de solidaridad y entre los que tampoco se presenta una identidad urbana claramente definida. Sobre esta base se efectúa la invasión con relativa facilidad en esta zona.
} 
mucha resistencia como en el caso de la segunda. Esto es, por ejemplo, claro en el caso de los migrantes. El primer síntoma de este tipo de invasión se percibe en el precio del suelo que empieza a decaer en el centro puesto que las características raciales y económicas de estos grupos (discriminados racialmente y con bajo nivel de vida), son distintos a los que habitan las zonas residenciales. La zona ocupada por estos grupos, regularmente migrantes pobres, entra en proceso de deterioro.

$\mathrm{Al}$ avanzar y consolidarse la invasión, se generan nuevas formas de asociación y competencia. Se habla de un momento climax de la invasión cuando la organización ecológica emergente alcanza un grado de equilibrio y estructuración que la hace inmune a invasiones sucesivas. En realidad, el carácter despiadado de la lucha competitiva tiene como finalidad imponer una cierta organización ecológica, como puede ser los usos del suelo en la ciudad; una vez efectuado este cometido, la competencia disminuye, emergiendo un orden moral específico que da un nuevo y vigoroso sentido a la solidaridad comunitaria e impidiendo de esta manera, al menos temporalmente, la entrada de nuevos invasores (ibidem).

En el interior de la comunidad cada formación ecológica funciona como una fuerza magnética colectiva que atrae y repele elementos según si éstos son deseados o no. Es esto, en realidad, lo que se halla en la base de la subdivisión biológica y cultural que existe en los habitantes de la ciudad. Esto es en fin lo que explica la formación de las áreas funcionales y la desigual distribución de los hombres en el espacio urbano.

La dimensión ecológica menor: las áreas naturales y el orden físico y moral

La conformación de las áreas naturales (uno de los conceptos clave en el enfoque ecologista) es el mecanismo por medio del cual se produce el espacio y la cultura urbana. Esto es analizado por Park tomando como objeto de análisis a los migrantes y a los grupos étnicos. En el estudio de estas comunidades Park observaba una segregación inicial proveniente del lenguaje y la cultura y, posteriormente, otro que descansa sobre bases étnicas. En el plano de la estructura social, tal y como lo plantea este autor, la segregación deviene movilidad social. De esta manera, en el conjunto de la sociedad y también en el interior de cada área natural, se llevan a cabo formas específicas de la segregación sobre la base de intereses profesionales, inteligencia y ambición personal. En 
esto se sustenta en realidad lo que se llama el mecanismo de autorregulación social mediante el cual, la sociedad, a través de la movilidad, selecciona a sus miembros más destacados, conformando simultáneamente las distintas áreas de asentamiento.

La creación de las áreas naturales implícitas en el crecimiento espontáneo de la ciudad da lugar a una estructura física y sobre ésta descansa una determinada estructura social y un orden moral específico. La estructura social es el resultado del proceso de movilidad mediante el cual los hombres cambian de estatus, ya sea ascendiendo o descendiendo de jerarquía. La estructura física o ecológica de la ciudad, por su parte, es un producto de los cambios ocupacionales y culturales expresados bajo la forma de cambios de localización. Es por esto que Park sostiene que: "La selección y la segregación social que crea los grupos naturales, determina también las áreas naturales de la ciudad." (Ibidem. ${ }^{6}$ Este mismo proceso de diferenciación que da lugar a una forma particular de ordenamiento territorial a través de la formación de áreas naturales origina la llamada división social del trabajo bajo su forma biológica (la estructura física o ecológica) que posibilita a la ciudad el desempeño de aquellas funciones que el crecimiento urbano hace imprescindible. Este fenómeno, en el esquema ecologista, es similar al que tiene lugar entre los organismos vivos, de tal manera que el papel de la división del trabajo en la ciudad y en la sociedad en su conjunto, es el de hacer posible el proceso de adaptación del hombre a su medio ambiente, de manera similar a la división del trabajo (división de funciones) que se presenta entre los organismos vivos a fin de realizar las funciones respiratorias, digestivas, etcétera.

El concepto de área natural antes mencionado posee un significado especial en la propuesta analítica de la escuela ecologista clásica porque, como lo ha hecho notar Saunders (op. cit.), permi-

\footnotetext{
${ }^{6}$ El concepto de área natural delimita, desde la perspectiva territorial, los fenómenos sociales de tal manera que se trasciendan las limitaciones de las áreas administrativas, que no siempre coinciden con los ámbitos en los cuales tienen lugar los procesos sociales. Éstos, como lo señala Wirth, no coinciden con los límites precisos de las divisiones administrativas. El crimen, las enfermedades, la desorganización familiar, etc., tienen una lógica propia de localización y ésta se encuentra estrechamente vinculada con el entorno ecológico; es decir, a determinadas áreas naturales (Wirth, 1964a: 183). Las áreas naturales surgen del proceso de expansión y de la evolución natural de toda ciudad. Estas áreas se conforman como una expresión del surgimiento de una infraestructura urbana regida por las redes de transporte, por la organización industrial y comercial, por el trazo de las calles, las características topográficas, etc. Las áreas naturales constituyen el producto espontáneo del crecimiento de las ciudades (Zorbaugh, 1968: 222-223).
} 
te confrontar el planteamiento teórico con un referente empírico concreto, como pueden ser los casos del Ghetto, los vecindarios, etc. Éstos, además de constituir un ámbito específico de la división del trabajo y un escenario particular de la cooperación competitiva (correspondiente al nivel de fenómenos de la comunidad) son, al mismo tiempo, un área moral (que corresponde al orden de cosas de la sociedad), humanizada como lo señala Park, por la cultura de sus habitantes, un sitio definido por el consenso y la comunicación. 'Representa, por tanto, un objeto, una 'cosa', la cual puede ser estudiada ecológica y sociológicamente, como una unidad natural o como una unidad social." (Ibidem.)

La escuela ecologista y el objeto de estudio de la sociología urbana hoy (conclusiones)

La presentación del marco conceptual elaborado por la escuela ecologista para dar cuenta de los procesos urbanos, tal y como se ha hecho en este documento, puede ser de utilidad para ubicar ciertaš rupturas y continuidades en la evolución del pensamiento social, al tiempo que se exponen algunas de las propuestas teóricas que se discuten actualmente.

En este contexto es importante establecer algunas precisiones y puntos de partida para ordenar las ideas al respecto. En primer lugar, es importante distinguir, por una parte, entre lo que son las formas espaciales, aquello que de alguna manera se concretiza en la ciudad; es decir (lo cual significa ya una forma de recorte teórico), el espacio construido en el cual ocurren determinados procesos de la vida social y los propios procesos sociales que, en apariencia, le dan contenido a esa dimensión del espacio constituida por su propia materialidad física. Esto es, aquello que, como ha señalado algún escritor, hace la diferencia entre el tablero de ajedrez y el movimiento de las piezas que en él ocurren. En segundo lugar, es pertinente establecer la propia diferencia o separación analítica de aquello que corresponde a la reflexión teórica, es decir, al objeto teórico que, aunque referido a los hechos de la vida social, tiene una existencia que no es reductible al llamado mundo de lo real. En síntesis, deben señalarse las diferencias que existen entre el orden de cosas que corresponden al dominio del ser, y aquellas propias del ámbito del pensar.

Esta acotación tiene dos propósitos. El primero es entender el sentido de la crítica de autores como Castells y Saunders a la pertinencia analítica de la sociología urbana, lo cual nos ubica en el nivel de fenómenos remitibles al pensar y el destino teórico reser- 
vado a ese nivel de la realidad constituido por los llamados procesos urbanos, lo cual nos ubica en el nivel de cosas que corresponden al ser. La crítica de Castells al recorte analítico de lo urbano no niega este orden del llamado concreto real, por lo que la ciudad como entidad física y lo urbano como proceso social sobreviven a su muerte teórica; siguen, por lo tanto, constituyendo una dimensión de la realidad que exige ser explicada, independientemente del sentido y la perspectiva analítica de esta explicación. En este sentido lo que está en tela de juicio en la discusión, en torno a las formas espaciales y a los procesos sociales, no es su estatuto ontológico sino su validez epistemológica.

El segundo propósito es ubicar algunas de las líneas de pensamiento en el campo de la sociología urbana (y quizás de las ciencias sociales en general) que han tratado de construir un objeto teórico con el fin de rescatar la especificidad de lo urbano dentro del conjunto de fenómenos que conforman los distintos niveles de lo real.

En términos generales y desechando derivaciones y variaciones específicas de los distintos planteamientos teóricos, pueden distinguirse dos grandes corrientes de pensamiento en el campo de la sociología urbana cuyos puntos de demarcación están en función del recorte analítico de su objeto de estudio y del objeto teórico que construyen. La primera perspectiva tiene que ver con aquellos enfoques de lo urbano en los cuales el objeto de estudio está constituido por lo espacial, bajo cualquiera de sus combinaciones posibles. Por ejemplo, lo espacial como estructura física construida por el hombre, lo espacial como determinante de conductas sociales, lo espacial como escenario de lo social pero como una realidad analítica relevante, lo espacial como una realidad socialmente producida, las condiciones sociales de la producción del espacio e, incluso, bajo la forma en que ha aparecido en la más moderna teoría sociológica de Giddens: lo espacial como elemento estructurante de lo social.

Esta línea de pensamiento, que legaliza al espacio como objeto de reflexión sociológica, emparenta a pensadores tan disímbolos como Park de la escuela ecologista clásica y Giddens (1972 y 1981), el más popular de los sociólogos actuales, pero incluye también las fecundas e imaginativas propuestas de Lefebvre $(1968,1970)$ y los trabajos de Harvey (1982) y Massey (1984).

La segunda perspectiva teórica tiene como principal característica su recorte analítico más estrictamente sociológico, porque ubica como objeto de reflexión lo social, más que lo espacial y los procesos urbanos, más que la ciudad como entidad física, pero sin atribuirle ningún estatuto de legalidad epistemológica a lo urbano 
que, bajo esta lógica, debe remitirse a la teoría social. En esta línea se ubican, tanto las primeras reflexiones de los clásicos (Marx, Weber y Durkheim), como las propuestas iniciales de Castells (y la llamada sociología urbana francesa) pasando, aunque parezca extraño, por la crítica culturalista que siguió a los esquem as biologicistas de la escuela ecologista clásica. Este último punto se encuentra sustentado en el hecho en el que, los culturalistas, establecen una cierta ruptura con las fuerzas naturales establecidas por el pensamiento ecologista como categorías explicativas de los fenómenos urbanos, sosteniendo el carácter social de estos fenómenos y reafirmando un orden de cosas que separa el mundo animal, de aquel que corresponde a lo social, aquello que en la obra de Levi-Strauss se establece como la frontera entre naturaleza y cultura.

Ahora bien, en el marco de este trabajo y ante la necesidad de ubicar a la escuela ecologista clásica, tanto en la evolución del pensamiento sociológico de lo urbano, como en la discusión más reciente, voy a centrarme fundamentalmente en la presentación de algunas ideas sobre la primera de las líneas de pensamiento que he señalado, tratando de establecer puntos de contacto, así como sus principales zonas de desencuentro.

Los planteamientos iniciales de los ecologistas no buscan propiamente construir un objeto de estudio para la sociología urbana, puesto que aspiran a elaborar una explicación general de la sociedad. Como sociólogos su campo de estudio estaba limitado a los fenómenos concretos, aquellos de la patología social que emergía del ámbito urbano. Como creadores de una ecología humana pretendían crear una metaciencia de lo social.

Ellos ven el orden urbano en el marco y como la confluencia de un conjunto de fuerzas naturales y como resultado de otras de carácter social; pero entre ellas, el mayor grado de eficacia corresponde a las del mundo natural, aun cuando ciertos elementos serían reductibles a la biología y otros a la sociología. La propuesta ecologista trata de tender el puente teórico entre ambas disciplinas, pero fracasa al rescatar el determinante de la causalidad natural en la construcción del orden urbano. En la visión ecologista la ciudad, aun cuando como sostiene Park se humaniza con la cultura de sus habitantes, se convierte en un factor explicativo que da cuenta de conductas sociales específicas. Las zonas de transición, el área de tugurios, para poner sólo algunos ejemplos, aparecen como decisivos en la generación de comportamientos patológicos.

El problema no resuelto por la escuela ecologista es, por lo tanto, el de la construcción de un objeto teórico de corte sociológi- 
co y, además, el de la separación entre el orden de cosas que corresponde al mundo de lo real y las que tienen que ver con la reconstrucción analítica de esa realidad; es decir, se confunde el objeto empírico y el objeto teórico. En este sentido, sus observaciones sobre lo urbano describen un tipo particular de sociedad (que corresponde a una etapa de desarrollo de la sociedad estadunidense) atribuyéndole un estatuto de universalidad.

La afirmación en el sentido de que no crean una teoría sociológica de la sociedad, ni una de la ciudad, tiene como base el hecho de que no delimitan una legalidad social para los fenómenos urbanos y sociales en general, en la medida que lo social es remitido a lo biológico y no explican, además, las leyes de funcionamiento y transformación del orden urbano bajo la perspectiva de su especificidad sociológica.

En cuanto a la confusión entre lo teórico y lo empírico, la crítica de Castells, por ejemplo, señala que esta escuela de pensamiento no supo trascender su problemática empírica o no pudo reconstruirla en un objeto teórico aplicable a una mayor generalidad de casos, y no pudo hacerlo porque no conceptualizó las leyes sociales que constituyen los fenómenos urbanos, describiendo a estos últimos bajo la forma de su representación alegórica. En este sentido el problema central no es tanto la validez de sus descubrimientos para explicar un orden de cosas en los casos específicos analizados, sino la aplicabilidad de sus hallazgos para otras situaciones con distintos procesos sociales. Toda teoría, como ha sido señalado (Zemelman, 1987) enfrenta la necesidad de su relativización, la cual proviene de los procesos históricos de los que surge y de los que da cuenta. El conocimiento, sostiene este autor, debe desarrollarse en una articulación histórica determinada para, de esta manera, explicar los procesos concretos, teorizándolos en función de su carácter histórico. Siguendo con esta argumentación, la razón fundamental de una ciencia, como es el caso de la ciencia social, no radica en sus instrumentos de verificación, sino en la construcción de una teoría; se trata, por tanto, de reconstruir el objeto de estudio, elaborar un objeto teórico que dé cuenta del referente empírico concreto. Es decir, la teoría se construye en la búsqueda lógica e histórica de las relaciones ąue subyacen y explican a los fenómenos, en la ubicación de aquello que es determinante, así como en las articulaciones de los distintos niveles en que se compone lo real (ibidem).

Los ecologistas no resuelven el problema, únicamente lo desvían, remitiéndolo a una esfera distinta de la realidad. Por ello en la discusión actual reaparece nuevamente y, otra vez en crisis el objeto teórico, tanto bajo su forma espacial como social, parece 
desvanecerse cada vez más. La cuestión central radica en la ubicación del espacio en la reproducción social y en la definición del orden de cosas de la realidad que justifique la existencia de una teoría sociológica de lo urbano ante la presencia, inoportuna a veces, de lo espacial cuya cualidad ontológica demanda una reflexión teórica.

Tal vez los viejos problemas detectados por los ecologistas surjan de nueva cuenta, pero lo hacen en un contexto teórico y epistemológico distinto. Aun dentro de estas versiones que rescatan la importancia de la dimensión espacial en la conformación de los procesos urbanos, éstos son remitidos a una legalidad social específica. Las diferencias son fundamentales y, aunque demarcan una etapa de crisis, también dan cuenta de un momento de madurez de las ciencias sociales, las cuales pretenden valerse de su propio instrumental analítico para explicar el nivel de fenómenos que le son propios. Así, por ejemplo, en los nuevos planteamientos de la realidad social en los cuales se retoma lo espacial, el problema a resolver ha dejado de ser el carácter social del espacio urbano, para tratar de explicarse la manera específica en que este espacio participa, no sólo como continente o soporte material de los procesos sociales, sino como elemento activo que influye en la estructuración misma de la realidad social. Autores como Lefebvre, Harvey y Giddens dan cuenta de una revaloración sociólogica del espacio en la teoría urbana y social en general.

Lefebvre, por ejemplo, ve al espacio como una realidad de múltiples dimensiones. Por una parte, lo considera integrante de las fuerzas productivas, puesto que puede asumir la forma de un medio de producción; pero también lo incluye dentro de las relaciones de producción en la medida que de él derivan formas de propiedad específicas. Por otra, es también un objeto de consumo, un instrumento para la dominación política y un elemento constituyente de la lucha de clases.

Bajo estas dos últimas acepciones (instrumento político y lucha de clases) la noción del espacio y del orden urbano de Lefebvre, aun cuando de carácter espacialista, se distingue de los ecologistas clásicos de manera rotunda. Los ecologistas veían, por ejemplo, la lucha por la obtención de posiciones en la estructura espacial y en la social como una lucha instintiva e individual, en la cual se expresaban las fuerzas naturales que rigen el destino humano, con el propósito de obtener ventajas económicas. Lefebvre, en cambio, ve al espacio urbano como una realidad que se ordena, homogeneiza y segrega por la acción del Estado para imponer su propia dominación y la de las clases que representa.

Para este autor el espacio, que el Estado y el capital han con- 
vertido en un valor de cambio, una mercancía con la cual se enajena y expropia la esencia humana (espacio abstracto), debe ser transformado en un espacio social, en el cual se manifieste la libertad individual, permitiendo al hombre romper con el mundo de la alienación que sustenta la reproducción material y moral de la sociedad capitalista. El espacio debe ser apropiado por quienes padecen su opresión, y debe ser transformado en espacio social (valor de uso) para hacer posible la completa liberación del hombre.

Giddens, por su parte, va más allá en la revaloración del espacio, puesto que lo considera como un elemento activo en la conformación del orden y la conducta social. Pero esto que aparentemente es una cercanía con la escuela ecologista es lo que más lo aleja de ella, porque el espacio que le interesa no es tanto el espacio físico como tal, sino aquello descrito (Coraggio, 1977) como la espacialidad de los procesos sociales, o como espacio social. El espacio no aparece como el momento alegórico de lo social, sino más bien como un elemento que interviene en su constitución.

Ciertamente la ciudad en Giddens no crece por las fuerzas inconscientes de carácter natural que impulsa a los hombres en el esquema ecologista, hay en su argumentación una clara dimensión social de los fenómenos urbanos que, según él, constituyen el centro de la moderna teoría social.

Giddens comparte con los ecologistas la constatación de una jerarquía en la estructuración del espacio que hace emerger determinadas conductas sociales, pero su argumentación no se sustenta en una causalidad extrasocial, puesto que el espacio asiste al momento de constitución de lo social y este mismo espacio que, en una de sus dimensiones, resulta de los actos rutinarios de la vida cotidiana, constituye una de las formas fundamentales de la cohesión social. El espacio más que escenario o determinante de lo social, es un elemento fundamental de la reproducción social.

Giddens señala dos niveles en los cuales participa lo espacial en la estructuración del orden social. Por una parte mediante la llamada "integración social" que da cuenta de los contactos directos entre los distintos actores del organismo social. En este sentido, los hombres llevan a cabo su vida cotidiana de una manera cíclica, de tal forma que la recurrencia permanente de sus actos, junto con la recurrencia también permanente de los actos de los otros, da lugar a un patrón espacial de conducta. Los hombres regionalizan las acciones de su vida cotidiana repitiendo determinados actos (ir al trabajo, viviendo diferencialmente los ámbitos de la casa, etc.) y esto se convierte en un elemento de cohesión social; los miembros de una comunidad no se plantean llevar a cabo una nueva actividad cada día, más bien la vida social se compone de 
actos que se repiten indefinidamente. Esta rutinización que origina la vida cotidiana genera una suerte de seguridad ontológica, una cierta fuerza que mantiene unidos a los hombres y que reproduce el orden social.

La otra integración que percibe este autor como elemento de integración, es "la integración sistema" mediante la cual se logra la cohesión entre personas que, aun cuando no se ven, aun cuando no establecen contacto físico directo, sostienen vínculos que los mantiene unidos. Bajo esta perspectiva la ciudad es considerada como un espacio de poder y dominación y esto ocurre así porque en ella se contienen los recursos que en verdad dominan la vida contemporánea, esto es, los llamados recursos distributivos (como es el caso de los productos que aseguran la subsistencia) y los llamados recursos de autoridad. Esto en realidad aparece como la explicación sustantiva del dominio que ejerce la ciudad sobre las áreas socioespaciales que le circundan; en ella, en fin, se concentra la fuerza y la inteligencia, tanto desde la perspectiva económica y política, como desde la militar (Saunders, 1984).

Algunas de las ideas de Giddens son compartidas también por Massey, sobre todo aquellas que aluden a las diferencias entre relaciones de clase que derivan de la influencia de los ámbitos espaciales específicos en las que éstas se presentan. Mediante esta argumentación se sostiene que las grandes tendencias de la sociedad capitalista no asumen las mismas características en los distintos países y regiones. Estas tendencias se modifican por la mediación de las coyunturas locales, las cuales le dan forma y contenido específico a los cambios globales.

De todas maneras esta consideración de las especificidades regionales en la conformación de procesos sociales particulares no soluciona el problema central, es decir, aquel que tiene que ver con el papel asignado al espacio en la génesis de lo social. Esto es así porque, cuando se hace entrar al espacio local como elemento modificador de las tendencias globales, en realidad no se está considerando al espacio en sí, sino a los procesos que ahí tienen lugar, de tal manera que se alude a la economía, a la política, a la cultura y a la historia en general de esas localidades, como los elementos que constituyen (aun cuando sea sobre una base espacial) las llamadas coyunturas locales o regionales; se está hablando, por lo tanto, de procesos sociales y no de procesos espaciales en la determinación de las coyunturas locales.

La aportación de Giddens, y esto lo distingue no sólo de los ecologistas, sino del resto de aquellos pensadores que he englobado dentro de la perspectiva espacialista de lo urbano, consiste en que no sólo enuncia el determinante espacial de los procesos so- 
cíales, sino que además expone las formas posibles de esta determinación y esto lo hace por medio de su argumentación de las formas de la integración social en las cuales el espacio se convierte en un elemento constitutivo de lo social y en la principal fuerza sobre la que se sustenta la cohesión social.

La llamada visión espacialista ha sido desdeñada en distintas corrientes de pensamiento por la presencia, como lo señala Giddens, de un cierto prurito sociológico. Esto ha impedido valorar aportaciones tan significativas dentro de esta corriente de pensamiento como las del propio Lefebvre y apreciar también la inmensa separación teórica de estos autores respecto a las primeras ideas esbozadas por los ecologistas y aun respecto a los clásicos del pensamiento social. Esta especie de sospecha espacialista cayó en su momento sobre autores como Harvey, quien trató de incluir algunos de los paradigmas ecologistas (como son los casos de la búsqueda de mejoras tecnológicas y de localización estratégica) dentro de un esquema de racionalidad económica, en donde el fin último es la maximización de la ganancia capitalista. Además de haber rescatado la importancia del espacio como medio directo para la reproducción capitalista en la medida que la inversión en el espacio construido, constituye la última alternativa de la producción capitalista para superar la caída de la tasa de beneficio.

La importancia de esta línea de pensamiento consiste en que eliminan la idea del espacio como una entidad pasiva sobre la cual los hombres hacen su historia y en el cual, además, transcurren los hechos de la vida en general, sino que, por el contrario, ven al espacio no sólo como el escenario de lo real, sino como un artífice de esta realidad. En esta corriente, por lo tanto, el espacio no es reductible al mundo de lo sensorial, y menos aún como producto de leyes naturales; por el contrario, es un elemento más de estructuración de la realidad social.

\section{Bibliografía}

Allihan, Milla (1988), "Estudios de comunidad y ecológicos", en Bassols, M., et al., Antología de Sociología Urbana, UNAM.

Anderson, Nels (1965), Urban Sociology, Karmatak University.

Bardo, John y John Hartman (1982), Urban Sociology, Peacok Publishers, Inc.

Bettin, Giafranco (1982), Los sociólogos de la ciudad, Gustavo Gilli, Barcelona.

Burgess, Ernest (1967), "The Growth of the City: an Introduction to a research Project", en R. Park, E. Burgess y Roderick McKenzie, The City, University of Chicago Press. 
Burgess, Ernest y Donald Bogue (1970), Urban Sociology, The University of Chicago Press.

Castells, M. (1978), La cuestión urbana, Siglo XXI Editores.

Giddens, Antony (1979), Central Problems in Social Theory, Macmillan. (1985), The Constitution of Society, Polity Press.

Gottdiener, M. (1985), The Social Production of Urban Space, University of Chicago Press.

Harvey, David (1982), The Limits to Capital, Basil Backwell.

Hatt, Paul y Albert Reiss (1957), Cities and Society. The Free Press, N.Y. Hawley, Amos [1950), Human Ecology, The Ronald Press Company.

Lefebvre, Henri (1978), El derecho a la ciudad, Peninsula, Barcelona. (1972), La revolución urbana, Alianza Editorial, Madrid.

Marcuse, H. (1972), Razón y revolución, Alianza Editorial.

Massey, D. [1984], Spatial Divisions of Labor: Social Structure and the Geography of Production, Macmillan.

McKenzie, Roderic (1967), "The Ecological Approach to the Study of the Human Community", en R. Park, E. Burgess y R. McKenzie, The City, The University of Chicago Press.

Park, Robert E. (1968), "The Urban Comunity as a Spatial Pattern and a Moral Order", en E. Burguess, The Urban Community, Greenwood Press Publishers, N.Y.

Park, Robert, Ernest Burguess y Roderick McKenzie (1967), The City, University of Chicago Press. (1952), Human Communities, New York, Free Press. (1988), "Ecologia humana", en Bassols et al., Antologia de Sociologia Urbana, UNAM.

(1968), "The city: suggestions for the investigation of human behavior in the urban environment", en R. Park, E. Burgess y Roderick McKenzie, The city, University of Chicago Press.

Parsons, Talcott (1961), "Some Considerations on the Theory of Social Change" en Rural Sociology, Xxvi, núm. 3.

Renter, E.B. (1968), "Sociology and Biology", en E. Burgess, The Urban Comunity, Greenwood Press, Publishers, N.Y.

Saunders, P. (1986), Social Theory and the Urban Question, Holmes and Meier Publishers, Inc., Nueva York.

Sutherland, E.H. (1968), "The biological and social processes", en E. Burgess, The Urban Comunity, Greenwood Press, Publishers, N.Y.

Wirth, Louis (1964a), "Human Ecology", en A. Reiss, Wirth on Cities and Social Life, The Chicago University Press. (1964b), "The scope and problems of the community", en A. Reiss, Louis Wirth on Cities and Social Life, The University of Chicago Press.

(1964c), "The Ghetto" y "Some Jewish Types of Personality", en A. Reiss, On Cities and Social Life, The University of Chicago Press.

Zemelman, Hugo (1987), "Razones para un debate epistemológico" en Revista Mexicana de Sociología, enero-marzo.

Zorbaugh, Harvey (1968), "The Natural Areas of the City", en Ernest Burgess, Urban Community, Greenwood Press Publishers. 